\title{
The distribution of NPC-1 protein in macrophages is altered after oxidized LDL lysosomal accumulation.
}

\author{
W. Gray (Jay) Jerome, Brian Cox, Jeremy B.Vaughan
}

Vanderbilt University Medical Center, Nashville TN 37232

Atherosclerosis (hardening of the arteries) remains a major cause of death in the United States. Massive cholesterol accumulation within macrophage lysosomes is a key event in the disease. The accumulation produces "foamy" appearing cells. The mechanism for this foam cell formation is unknown. In previous studies we showed that normal intracellular cholesterol transport is inhibited when cholesterol accumulation in lysosomes is stimulated by oxidized LDL (oxLDL). We continue our analysis of transport inhibition in the current studies.

Little is known about the regulation of intracellular cholesterol movement except that it occurs primarily through vesicular transport. One key integral membrane protein has been identified. This protein, NPC-1, is mutated in Niemann-Pick type C (NP-C) disease. In this disease cholesterol accumulates within lysosomes because it is not transported out. NP-C cells also show an altered distribution of the mutated NPC-1 protein. Whereas normal cells show a relatively uniform distribution of NPC-1 in ER, Golgi, and lysosomal membranes, Niemann-Pick type C cells have a higher concentration of NPC-1 within the Golgi apparatus.

Because of the similarities between cells from patients with NPC and the foamy macrophages of atherosclerosis, we investigated the distribution of NPC-1 protein in foam cells. THP-1 macrophages in culture were incubated with either oxLDL or acetylated LDL (acLDL). OxLDL produces lysosomal cholesterol accumulation. AcLDL, on the other hand, produces equivalent levels of cellular cholesterol but it accumulates as cholesteryl esters within the cytoplasm. Thus oxLDL treatment mimics atherosclerotic foam cells and acLDL treatment provides a control. Cells were grown in culture wells attached to microscope slides and incubated for three days with lipoprotein. Cholesterol values more than doubled in cells incubated with lipoprotein. NPC-1 protein was localized in cultured cells using a two-step immunofluorescence technique with Alexa-488 as the fluorescent tag. The NPC-1 distribution was compared to that of Golgin 97 (a Golgi membrane marker) using a similar method but with Texas Red as the fluorophore.

Before incubation with lipoprotein, NPC-1 fluorescence was weak and not concentrated in any specific region of the cell. Treatment of cells for three days with acLDL did not change this pattern. However, treatment of cells for three days with oxLDL produced a distinct perinuclear distribution of NPC-1 protein that colocalized with the Golgi marker (Figure). Eighty percent of the Golgi marker overlapped with NPC-1 fluorescence and 20\% of the NPC-1 fluorescence was found in the Golgi region. In contrast less than 5\% overlap was seen between the Golgi marker and NPC-1 fluorescence in acLDL-treated or control cells.

These studies show that oxLDL produces an accumulation of NPC-1 protein in the Golgi apparatus. The accumulation appears linked to the inability of the cells to remove lysosomal cholesterol. Since a similar redistribution in NPC-1 patients has been linked to defective transport of cholesterol, we 
hypothesize that the lysosomal cholesterol accumulation seen in atherosclerosis may be related to a similar trafficking defect.

The authors gratefully acknowledge Dr. Y. A. Ioannou for providing the anti-NPC-1 antibody.

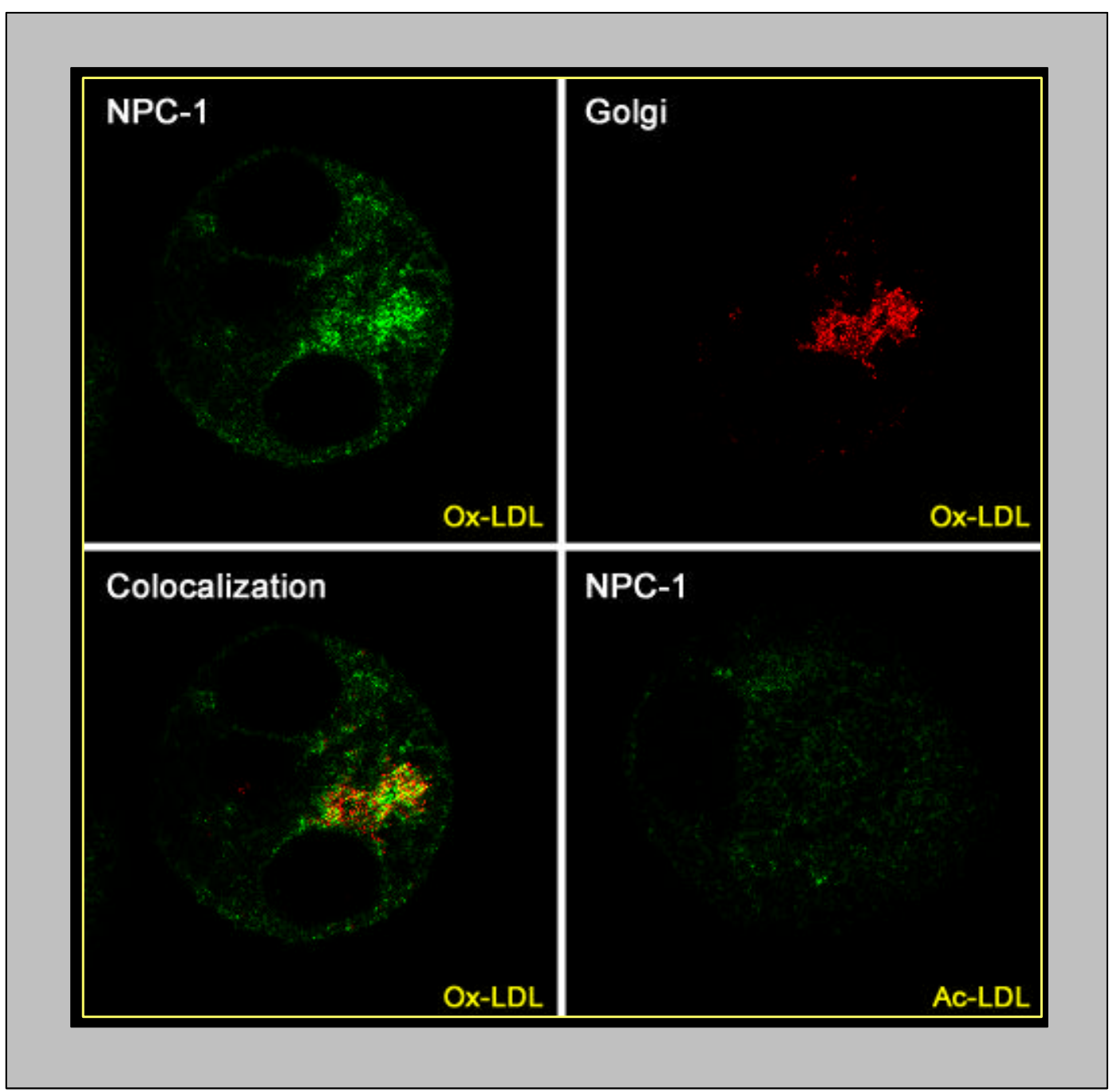

Figure: THP-1 macrophages incubated with either Ox-LDL for 3 days. NPC-1 protein fluorescence is seen as green in the top left panel. Golgi membrane fluorescence is seen in red (top right). Colocalization of the two patterns occurs as yellow when the two images are superimposed (lower left). Incubation of cells with Ac-LDL (lower right) did not produce a concentration of NPC-1 protein. 